\title{
Grotesque et ironie dans le théâtre de Jean-Marie Piemme
}

Au théâtre, le genre comique ne va pas de différence des modèles reconnus, l'arrière-fond soi. Il est généralement associé à un répertoire dans lequel s'enracine le comique est ici le aux effets attendus, proche du cabaret et souvent monde politique, qui dès lors occupe lié au secteur commercial. En Belgique structuralement la place de la sphère privée francophone, les dramaturgies qui émergent dans bourgeoise. Très vite, l'usage du grotesque les années 1980 et qui s'imposent aujourd'hui oriente le texte vers la farce et lui confère un se sont développées dans un contexte de crise effet dévastateur que ne pouvait avoir le économique et de reflux des élans vaudeville. On retrouve, essentiellement révolutionnaires et utopiques. Et, à l'exception manifestés par le Premier Ministre, l'inversion des spectacles d'Yves Hunstad relevant d'un du haut et du bas, la scatologie, le sexe et la comique quasi métaphysique (mais sous la forme trivialité qui composent l'excès carnavalesque à du one man show), les pièces de Jean Louvet, la manière rabelaisienne. Mais chez Piemme, il les premières pièces de Michèle Fabien, de Paul ne s'agit pas d'un débridement salutaire ni d'une Émond, de Jean-Marie Piemme et les premières explosion vitale. Au contraire, le dramaturge créations du Groupov relèvent d'un « sérieux » présente ce comportement comme la norme de incompatible avec toute forme d'humour. ses personnages dans leur face privée.

Cependant, chez Piemme, à mesure que Corrélativement, ce sont leurs discours publics, s'affirme son écriture pour le théâtre, l'ironie se ceux qui concernent le monde social, qui fait de plus en plus prégnante. Mais, loin d'être paraissent totalement fallacieux. Le grotesque déclinée comme une modalité d'écriture unique, constitue ici l'unique dimension des elle s'institue en contrepoint et vient relayer la personnages, qu'il rend de ce fait monolithiques. dérision ou le grotesque. Une telle hétérogénéité Il représente, en somme, un moment de pure n'a rien d'aléatoire et tend, au contraire, à négation et tire ce texte vers la satire.

s'organiser en une véritable articulation.

Dans la pièce suivante, Commerce

Dès sa deuxième fiction, Sans mentir ${ }^{1}$, gourmand ${ }^{2}$, les traces de grotesque ont Piemme se propose d'explorer le genre comique, pratiquement disparu, laissant place à la dérision, dont l'archétype, pour notre tradition, reste le chez certains personnages uniquement. Ainsi, vaudeville. Il reprend donc les codes, les face à ceux qui mènent une lutte, assez personnages-types et les situations de ce genre désespérée il est vrai, pour trouver un sens et place un premier ministre, sa femme et son possible à leur existence dans le climat très noir chef de cabinet dans le huis-clos d'une chambre d'une société mercantile, un personnage comme d'hôtel de province et en situation de commettre Benny abandonne très tôt la partie dans une tromperies et usurpations. Toutefois, à la tentative d'oubli de soi et du monde qui n'est

1 Piemme (Jean-Marie), Sans mentir. Paris, Actes Sud-Papiers, 1989. Création en mars 1989 au Centre théâtral de Namur dans une mise en scène de Bernard Debroux.

2 Piemme (Jean-Marie), Commerce gourmand. Paris, Actes Sud-Papiers, 1991. Création en 1991 à la Rose des Vents de Villeneuve d'Ascq dans une mise en scène de Philippe Sireuil. 
pas sans évoquer les créatures de Beckett. Or, réévaluation critique des personnages, l'Homme élaborant, à l'instar de ce dernier, une et la comédienne, qui, au final, opère pour dramaturgie centrée sur le personnage, Piemme chacun des deux, en sens opposés. Si l'Homme ne sature pas son univers théâtral de dérision et apparaît moins dérisoire grâce à ce bagage laisse advenir des contrepoints.

Ainsi, dans Le Badge de Lénine ${ }^{3}$, l'Homme n'en finit pas de se narrer sur un plateau de théâtre quasi désert. Il a comme seul auditoire une actrice restée pour répéter son texte et, fort de cette audience, il livre des souvenirs anecdotiques entrecoupés de dialogues avec la jeune femme. Mais, si les bribes d'histoires qu'il débite sont censées construire son identité, le personnage apparaît très vite assoiffé de ses propres mots : sa logorrhée devient à la fois une modalité et une attestation d'existence. Cet homme, nous dit Piemme en une sorte de clin d'œil, se leurre largement. Le dessein qu'il assigne à son monologue, voyage immobile dans l'espace clos du théâtre, en fait un être dérisoire.
Mais le dramaturge ne s'en tient pas à la composition d'un personnage monolithique qui s'ajusterait à un monde désenchanté et privé de sens. Le huis-clos du théâtre, qui détermine puissamment la structure de la fiction, ne retire pas l'individu du monde social. Presque à l'inverse de la dramaturgie beckettienne qui ménage toujours peu ou prou une place au tragique en menant jusqu'au paroxysme un «Moi » isolé de toute société, l'individu mis en scène par Piemme est énorme de sa sociabilité inéluctable. Comme chez Beckett ou Ionesco, l'auto-dérision est chargée d'un effet dévastateur, mais chez Ionesco, la dérision s'applique à tout (vie privée, langage, société...), tandis que chez Beckett, elle postule le refus de toute signification pour et par l'œuvre à tout le moins. Piemme, lui, en limite davantage l'effet. Certes, il compose, pour son personnage, le double point de vue de celui qui à la fois vit et se regarde vivre. En faisant surgir un aspect " plus savant » des discours apparemment anecdotiques de l'Homme, l'auteur le conduit à sortir de son jeu et partant, à rendre manifestes les codes et la forme du jeu. Jeu théâtral, bien sûr, qui offre aux metteurs en scène un espace jubilatoire d'expérimentation des mises en abîme. Mais la convocation de références culturelles (Mozart, Shakespeare, Lénine...) en contrepoint à la s'appuie la pièce. Ainsi, il se replace à une même dérision et à l'anecdotique provoque une distance de ses personnages et des spectateurs,

3 Piemme (Jean-Marie), Le Badge de Lénine. Paris, Actes Sud-Papiers, 1992. Création en 1992 au Théâtre Varia dans une mise en scène de Philippe Sireuil.

4 Piemme (Jean-Marie), Le Badge de Lénine, op. cit., p. 49. 
les entraînant les uns comme les autres à se mettre des discours s'arrime aux contradictions de en action. Les personnages ici ne portent pas la l'autre pour se développer jusque dans un excès vision d'un univers dérisoire et d'un monde où surgit l'ironie. L'échange direct et frontal impossible et le public ne reçoit pas passivement n'est contradictoire qu'à un premier degré. Car, la provocation que constituent l'échec et l'aporie clé de l'effet ironique, les propos se mettent à de toute fable. Restant dans une sorte de retrait, distance, atteignent un second degré et l'auteur, par l'ironie, prend néanmoins une l'ensemble du texte se trouve plongé dans la position implicite. Si celle-ci n'indique guère de satire sans fond, sans vérité masquée, et qui serait voie, elle suppose une forme de jugement qui adressée, elle, au lecteur-spectateur. Telle est, en demande à être explicitée.

C'est que le dramaturge traite la langue prête à un jugement sans critères bien définis. compte tenu de son usage social. À rebours des Position de surplomb peut-être, mais qui pose pratiques consacrées par l'orthodoxie littéraire, un défi.

il ne la présente pas comme un objet autonome, On voit combien ce projet dramaturgique se une pure forme, ni comme instrument de fonde sur la distance, la dissociation et le médiation. Il la façonne plutôt pour la libérer désaccord plutôt que sur l'empathie et la autant que possible, des codes établis. Ainsi le compassion. Plus particulièrement, il donne lieu langage dont usent les personnages ne renvoie à une satire des simulacres qui soustraient toute pas systématiquement à la position que ces une partie du réel à la saisie intellectuelle et derniers occupent dans le monde social. créent ainsi l'apparence d'un autre monde, plus Parallèlement s'érode leur adhésion au jeu même policé et vecteur de passivité.

engagé sur scène. Dans cette forme de distanciation, les mots ne disent pas plus le personnage qu'ils ne visent à convaincre la salle.

Au début des années 1990, Piemme diffuse ainsi, sous forme de tapuscrits, plusieurs textes repris sous le titre Ciel et simulacre. L'ensemble

L'écriture orchestre donc une circulation ne sera publié qu'en $2000^{7}$, et passablement triangulaire entre le langage, les personnages et remanié : certains textes sont raccourcis, tandis le public qui sera développée à l'extrême dans que d'autres ont disparu. En dépit de ces Toréadors ${ }^{5}$. La pièce met en scène deux hommes dans un salon lavoir. Le gérant, Momo, immigré du sud, y confronte ses opinions politiques à celles de Ferdinand, immigré russe, ex-cadre d'entreprise, à présent clochardisé. Tout le dialogue prend ici un caractère factice et et de Kleist. Si Voltaire tient ici lieu d'emblème s'apparente plutôt à une joute oratoire. Le débat quant à l'ironie qui parcourt le théâtre de rebondit, comme défilent les sujets d'actualité Piemme, Kleist représente la grande figure ou les problématiques sociales que les tragique d'un écorché en perpétuelle errance. Le personnages véhiculent : l'intégration de texte l'oppose à Goethe comme en un match où l'immigré et la lutte acharnée pour se faire une se disputent visions du monde et esthétiques. place, quitte à « enfoncer » un autre - « dans le Mais, au-delà de ce débat d'école parodié, la monde de la survie, ton frère est aussi ton mise en situation des personnages vient ennemi ${ }^{6}$. Et puis Momo finit par employer interroger ce qu'il reste aujourd'hui de ces Ferdinand dans son lavoir et Ferdinand par trahir grandes attitudes. Présentés comme des Momo. L'échange de propos, vif et rythmé, personnages de foire, Goethe et Kleist s'étiolent délivre d'abord un humour ravageur: toute la comme symboles dans un monde où le perspective sociale (où se reconnaît la Belgique divertissement est devenu une valeur première. et où le linge sale devient métaphore) est C'est le sens de la didascalie qui clôt la deuxième emportée dans le flux. Plus précisément, chacun «pièce ${ }^{8}$ :

5 Piemme (Jean-Marie), Toréadors. Carnières-Morlanwelz, Éditions Lansman, 1999. Création en 1999 au Théâtre Le Public dans une mise en scène de Philippe Sireuil.

6 Piemme (Jean-Marie), Toréadors, op. cit., p. 47.

7 Piemme (Jean-Marie), Ciel et simulacre. Carnières-Morlanwelz, Éditions Lansman, 2000.

8 S'il faut d'emblée exclure la notion de « scène ", celle de tableau ne s'applique pas davantage à l'ensemble Ciel et simulacre dans la mesure où aucun élément n'établit de lien, si ce n'est une convergence de propos. 
Kleist jette un caillou sur Goethe. sociales s'énoncent explicitement dans le texte, Rumeur d'une foule en liesse. L'acteur prises en charge par les personnages eux-mêmes, Goethe et l'acteur Kleist se replacent comme signe, en apparence, d'une grande lucidité. Les au début, visages dans les portraits. Le peuple riches et les pauvres s'opposent, les unes entre. En riant, il jette des tartes à la crème revendiquent les attributs du luxe (« Je suis sur Goethe et sur Kleist. ${ }^{9}$

Andrée, celle qui aime les fourrures $\left.{ }^{11} »\right)$, les autres, leurs dispositions sociales («Entrée à

Sous la double enseigne de Benjamin et de l'école, sortie de l'école, je n'ai rien appris à Baudrillard, Piemme montre ici, comme dans Le l'école [...] Je suis Véra celle qui dit non $\left.{ }^{12} »\right)$ Badge de Lénine mais en accentuant les tandis qu'une troisième énonce son statut dimensions satirique et parodique, comment la d'immigrée. Un tel marquage sociologique valeur d'usage recouvre le champ du symbolique, tandis que l'aura disparaît.

De même, le mythe du divertissement à tout prix sous-tend la pièce $O n$ dirait des vrais où l'auteur mêle en une vaste métaphore, le spectacle du genre reality-show et le grand commerce : mêmes moyens, mêmes objectifs. Piemme enferme ses personnages sous la coupole oppressante d'un centre commercial, image d'un monde de la consommation, en apparence vaste et ouvert ( « le grand théâtre du monde »). Mais ce qui s'y joue perd son poids de réel, rythmé par les sifflets de Bogbog qui annoncent la parade. Sorte de grand animateur, Bogbog orchestre la vie : il édicte les valeurs "Chaque âge de l'Humanité réclame de nouvelles illusions " ${ }^{10}-$, il engage les acteurs, siffle la fin de la scène, galvanise en somme le show. Mais ce monde découvre peu à peu un huis-clos où la présence du vigile incarne l'ordre et où, en patron d'entreprise aux allures méphistophéliques, Bogbog récupère et réintègre, dans son discours publicitaire, toute parole et toute attitude diluante pour l'ordre social qu'il relaie. Si le dramaturge fait planer sur son texte l'ombre du grand capitalisme incarné par les États-Unis, l'attention se déporte pourtant sur le jeu du visible et de l'invisible que met en œuvre le simulacre. Dans ce monde de la « joie de vivre ", où " rien qui ne s'achète et qui ne se vende ", l'instance même du pouvoir se fait invisible et les principes qui président aux visions et aux divisions du monde paraissent, dès lors, « normaux » et « naturels ».

(« C'est quoi une bonne patronne ? Quelqu'un qui t'apprend l'injustice $\left.{ }^{13} »\right)$, qui s'est précisé lors du remaniement des textes, ne produit pourtant aucun mouvement subversif profond. Si la position sociale des personnages génère des luttes et si les rapports de domination stimulent les instincts, jamais l'adhésion au jeu du « grand théâtre du monde » ne sera remise en question. Aucun des personnages ne voit le piège dans lequel il est pris, cette cloche transparente délimitée par le vigile et Bogbog, et qui enserre leur liberté. Si le vigile est éliminé dans une phase de révolte d'un des personnages contre l'oppresseur, Bogbog garde intact un pouvoir occulté par sa manipulation et sa séduction. L'aveuglement des individus reste donc complet et les fige inexorablement dans des rôles qu'ils ne pensent pas quitter. Tous répondent au coup de sifflet de Bogbog pour la parade finale.

Parodie des shows médiatiques, le texte en épouse à certains moments les formes, créant ainsi une alternance propice à la mise à distance. Cette transformation caricaturale, si elle renoue avec des procédés populaires, est requise ici pour dénoncer une pratique populaire ou plutôt massmédiatique. Mais c'est par l'ironie que la satire sociale prend toute sa dimension et que se dessinent en filigrane les contre-valeurs. L'auteur sous-entend en effet que la lutte se trompe de cible, qui abat les gardiens de l'ordre sans même apercevoir les contours et les formes du système qui asservit dans une joyeuse unanimité. En somme, il élabore une parabole - ce genre à « double fond ", à la fois fable et «leçon»

On dirait des vrais met cependant en scène comme le qualifie Patrice Pavis ${ }^{14}$ - permettant des personnages féminins dont les positions aux spectateurs d'apercevoir l'aspect

\footnotetext{
9 Piemme (Jean-Marie), Ciel et simulacre, op. cit., p. 14.

10 Piemme (Jean-Marie), Ciel et simulacre, op. cit., p. 28.

11 Piemme (Jean-Marie), Ciel et simulacre, op. cit., p. 15.

12 Piemme (Jean-Marie), Ciel et simulacre, op. cit., p. 15.

13 Piemme (Jean-Marie), Ciel et simulacre, op. cit., p. 18.
}

14 «La parabole est un genre à "double fond" : le plan de l'anecdote, de la fable, utilise un récit facilement compréhensible et plaisamment raconté qui est actualisé dans l'espace et le temps, il évoque un milieu fictif 
concentrationnaire de cette coupole transparente qui enceint la socialité ${ }^{15}$. Notons au passage que ces contre-valeurs sur lesquelles s' appuie l'ironie ne sont décryptables que par un public déjà doté de certaines compétences culturelles. Public en général susceptible d'avoir relativisé et dénié cet univers médiatique triomphant. En quoi l'inversion - par rapport à l'usage littéraire reconnu - qui consiste à recourir à une forme marquée comme populaire pour dénoncer une pratique de masse, tend à annuler son impact. En effet, si la parodie vise à briser l'autorité de l'objet parodié, elle n'opère pleinement que sur un récepteur convaincu de ce statut d'autorité.

La quatrième " pièce ", La Vie Luna Park, débride plus encore la satire de la séduction d'un monde lisse et pailleté. Piemme y montre des personnages pétris de suffisance, sortes de nouveaux riches maniant une langue de bois adaptée à leur condition, mais aux ordres de l'Oncle Sam, dont le nom ne laisse planer aucune équivoque. Enfin, exacerbant encore la dénonciation à la source de la satire, la dernière "pièce », Printemps de la bêtise, met en scène un show télévisé où le candidat répond à des questions de culture générale. À l'exception de celles liées au sport, ses réponses trahissent, plus que l'ignorance, l'indifférence aux enjeux politiques de la mémoire et soulignent la désintégration de l'esprit critique. Et si la mémoire historique manque totalement au candidat, la transposition théâtrale du show médiatique fait également surgir le caractère aléatoire de ce savoir décontextualisé qui, parce qu'il n'est relié à rien, ne renvoie qu'à lui-même et devient aussi inerte qu'un objet. La critique des médias, esquissée de la sorte, induit un lien entre la façon dont l'audiovisuel donne accès au monde et la perte d'esprit critique :

CANDIDAT : Je regarde souvent les infos, je
passe d'une chaîne à l'autre, ce qu'on
voit... je ne suis pas d'accord.
Présentateur : Avec quoi ?

CANDIDAT : Avec ce qu'on voit.

PRÉSENTATEur : C'est-à-dire?

CANDIDAT : Je ne sais pas. Foutez-moi la paix avec vos salades ${ }^{16}$.

Avec Ciel et simulacre, Piemme, sans user de détours, projette à la face des spectateurs ce qu'il montre et dénonce. Se refusant ici à envisager les mécanismes et les processus, il tend un miroir grossissant où la satire se porte sur un mode de société, résumé par les références à l'Amérique capitaliste. L'ironie, quant à elle, s'exerce à partir de comportements stéréotypés : la nouvelle riche, l'immigrée crédule, la révoltée... Sous le sarcasme, il faut dès lors déceler les marques d'une critique acerbe et incisive des schèmes de perception dominants. Derrière les formes de l'adhésion, commandées par la consommation et un certain monde audiovisuel, l'auteur n'a de cesse de débusquer l'acceptation, voire la revendication, d'une indigence intellectuelle. Ce faisant, il porte à la lumière cet accord tacite au monde qui rend possible toutes les dominations.

Comme le montrent ces quelques exemples, l'ironie constitue la clé de voûte du théâtre de Jean-Marie Piemme et sans doute est-ce par elle que, paradoxalement, cette œuvre gagne toute son autonomie. Par elle, cette écriture s'écarte de la dimension encore transitive du langage quand il s'agit de dire la perte ou, à l'extrême, le rien. À cet égard, il est intéressant de constater que lorsque deux compagnies congolaises s'emparent de Toréadors ${ }^{17}$, elles tirent le texte vers la satire, en l'occurrence du pouvoir étatique de leur pays, un peu au détriment de l'ironie de l'auteur.

Cependant, lorsqu'à la fin des années 1990, Piemme écrit une pièce plus directement inspirée du contexte sociopolitique, il libère une autre modalité du discours ludique. Dans Café des patriotes $^{18}$, il montre, sur l'arrière-fond de

ou réel où les événements sont censés s'être produits ; le plan de la "morale" ou de la leçon est celui de la transposition intellectuelle, morale ou théorique de la fable. C'est à ce niveau profond et "sérieux" que nous saisissons la portée didactique de la pièce et que nous pouvons, le cas échéant, la mettre en parallèle avec notre situation présente. », (PAVIS (Patrice), Dictionnaire du théâtre. Paris, Armand Colin, 2002).

15 « Là où le réel était donné en tant qu'objet brut, le social présuppose un minimum de traitement ou de construction. » (Dubors Jacques, Les Romanciers du réel. De Balzac à Simenon. Paris, Seuil, coll. Points/ Essais, 2000, p. 42).

16 Piemme (Jean-Marie), Ciel et simulacre, op. cit., p. 43.

17 Le Marabout Théâtre et l'Écurie Maloba dans une mise en scène de Nzey Van Musala en 2004.

18 Piemme (Jean-Marie), Café des patriotes. Bruxelles, publications de l'Ensemble Théâtral Mobile, coll. Didascalies, 1998. Création en 1998 au Théâtre Varia dans une mise en scène de Philippe Sireuil. 
tueries et de réseaux politico-mafieux, débordement la valeur régénératrice que l'ascension d'un cafetier. Le huis-clos du café Bakhtine attribue au grotesque rabelaisien, des patriotes permet d'organiser une géographie Piemme ne fait pas davantage de Freddy un être des comportements en fonction de l'attraction mauvais ni démoniaque. On observe ici encore qu'exerce Willy Dewolf, patron de café devenu, que ce n'est pas le monde qui serait devenu à la fin de la pièce, sénateur d'extrême-droite. étranger ou vide. Le grotesque, en effet, se porte Mais pour mettre en lumière les mécanismes sur un type de personnel précis et ne participe d'extrême-droite, Piemme ne s'en tient pas aux donc pas d'une vue quelque peu globalisante sur déterminants extérieurs communément le monde et les hommes. Lié à des individus et mobilisés. Dénudant ses personnages pour en non à des situations, il insiste sur la coupure entre montrer la trame, il fait voir l'articulation du les êtres, à rebours des utopies de la fraternité et corps subjectif au monde social. C'est alors, de la communauté. Partant, il relance aussi le qu'insistant sur la corporéité de toute vision du questionnement sur le sens et sur l'agir : que monde, il recourt une fois encore au grotesque. faire, comment faire avec de tels êtres ? Face à Et à nouveau, le grotesque semble masquer un l'attitude de Freddy, les deux femmes qui s'y malaise quant au politique, comme si confronté confrontent adoptent des réponses différentes : à une aporie, l'auteur contre-attaquait l'une, Carmen, la compagne de Dewolf, prend immédiatement, en poussant les processus qu'il Freddy à son propre piège du chantage et rétablit observe jusqu'à l'énorme, jusqu'à leur un rapport de force où elle domine; l'autre, paroxysme :

Claudia, tente de faire jouer sa supériorité intellectuelle et se fait frapper.

FREDDY : Il a encore dit : « cette femme-là Immanquablement, des êtres comme Freddy ne est une princesse. [...] Donc, tu dois être quittent pas leur terrain de « jeu » et toute comme un chien. L'aimer comme un tentative pour les en déloger s'avère vaine. C'est chien. $[\ldots]$ »

CARMEN : Il est très gentil Willy. que le rapport aux autres tel qu'il est vécu par le personnage s'enracine dans le ressentiment qui

FREDDY : Donc je suis le chien et tu es la maîtresse.

CARMEN : Et alors?

FREDDY : Alors l'équation est assez simple : on le fait ou on le fait pas.

CARMEN : On fait quoi ?

commande une recherche d'identité sociale : « J'en ai assez de suer en coulisse! Je ne veux plus brosser le caca de personne. Je viens d'en bas mais j'ai des ailes, je suis un golfeur, je suis un swingueur. » 20

Avec la construction de ce type d'individu,

FREDDY : Je suis monté comme un gorille. Je Piemme met au jour l'ancrage quasi intime de dis ça juste parce que c'est la vérité ! Je comportements d'extrême-droite et laisse ainsi les ai eues toutes ! [...]

CARMEN : Arrière, babouin.

FREDDY : Embrasse, écarte.

CARMEN : Il faut une raison pour faire les choses. Quelque chose qui vive ! Toi, tu n'as que des habitudes, tu baises par habitude, rien que ta mécanique qui part toute seule.

FREDDY : Connasse !

CARMEN : Tu es un minable, Freddy, et tu ne le sais même pas ${ }^{19}$.

Dans l'inversion du haut et du bas, dans la produit un effet de réel sans que l'auteur focalisation exclusive sur le sexuel, et même n'intervienne sur la matière, travaillant plutôt sur dans l'automatisme, on retrouve des schèmes qui le cadrage et la distance. Le grotesque vient font de Freddy, l'homme à tout faire de Dewolf, pointer ce qui parait irréductible et qui, de l'ordre un personnage grotesque. Sans donner au du pulsionnel, produit des effets sociaux.

19 Piemme (Jean-Marie), Café des patriotes, op. cit., pp. 168-170.

20 Piemme (Jean-Marie), Café des patriotes, op. cit., p. 170. 
On ne peut dès lors l'envisager dans l'optique spécifiquement du sens du social. Mais si d'un monde incohérent ou privé de sens. Au l'auteur peut faire voir la trame qui institue ces contraire, l'auteur pointe, non le monde, mais personnages comme des êtres avant tout sociaux, une manière d'être au monde. Surtout, il invite il semble n'indiquer aucune possibilité d'en à considérer l'hétérogénéité, la pluralité, moins neutraliser l'action négative. Le caractère anticomme une perte ou un déclin tragiques, mais dialectique du grotesque ne permet guère de comme une modalité du social. En ce sens, le dépasser le constat. Et l'on pourrait ici se grotesque serait alors le lieu d'une critique plus souvenir, par exemple, de ces intellectuels juifs féroce. Non dialectique, il brise et casse net, allemands s'attachant à voir et à montrer Hitler forçant la réaction ou la riposte, celle des autres comme un bouffon à défaut de démonter les personnages, mais aussi celle du spectateur. Il a mécanismes de l'ascension du nazisme comme en fait retrouvé une cible, là où la dérision s'y essaiera Brecht dans La Résistible Ascension entraînait à l'auto-mutilation et restait de l'ordre d'Arturo Ui. Une fois la cible dévoilée, Piemme du constat ou de la dénégation. Le grotesque chez suggère-t-il de réinstaller un rapport de forces ? Piemme est une attaque (Freddy cogne) qui oblige l'adversaire à se repositionner.

Aussi dans ce théâtre, le grotesque ne prêtet-il pas nécessairement à rire. Il n'est pas convoqué en soi et pour soi, mais désigne une manière d'être au monde qui recèle un potentiel de coercition : dans Café des patriotes, Freddy veut asservir les femmes à ses pulsions sexuelles, Dewolf veut recréer un ordre social fort, tandis que, dans Printemps de la bêtise, le candidat au jeu de la télévision donne toute latitude à ce médium pour déployer sa puissance d'aliénation. Méthode pour faire voir ce qui est à combattre, le grotesque, on l'a dit, semble cependant irréductible. En le convoquant, l'auteur laisse aussi voir qu'il n'a pas les moyens de le dépasser.

" Mais le peut-on? " semble alors demander l'ironiste au spectateur. Et en dissociant le grotesque du tragique - alliance quasi canonique que l'on trouve déjà chez Hugo -, il incite à effacer de l'horizon mental le cadre d'une unité perdue. Dans cette optique, le personnage grotesque dont l'auteur ne cesse de mettre en lumière l'assise pulsionnelle n'incarne pas une monstruosité, une nature particulière venues noyauter le monde social. Il est aussi $d u$ monde et nous ne pouvons l'en retrancher au nom d'une quelconque supériorité intellectuelle ou morale. Mais nous ne pouvons pas davantage le réduire ni l'assimiler. Or, si le grotesque inquiète, c'est qu'il est ici dangereux et qu'il menace : Freddy se montre violent et Dewolf veut orienter le fonctionnement de la société vers un ordre égocentriste et liberticide.

Cependant, ces personnages étant une production, une émanation, du social, ils doivent tant bien que mal être compréhensibles. Voici donc le grotesque et la dérision réintroduits par fondements.

Piemme dans la perspective du sens, et plus négliger : lorsque Claudia est agressée par Freddy, un homme intervient, frappe et met en fuite l'agresseur et, presque à la fin du texte, Carmen «balançant "ses vérités à Dewolf semble bien prendre l'ascendant toute crainte évanouie... Mais ce ne sont là, somme toute, que des réponses anecdotiques dans la fiction dont elles ne font que relancer le cours ou le clore. Au final, le défi revient dans le camp des spectateurs. Mais il a été encadré par l'ironie de l'auteur.

C'est que, précisément, la plupart des pièces de Piemme s'opposent à la doxa qui tendrait à purger la société de ces êtres malfaisants pour enfin retrouver un monde de bonté, pur et originel. Contre le leurre utopique d'un monde idéal perdu, l'auteur compose un patchwork social où tous les dualismes se dissolvent joyeusement en une vaste intrication lovée au cœur de chacun des personnages. C'est dès lors en sortant ces êtres grotesques tout empreints de négativité de leur statut d'exception que les combattre s'avère possible. Plus efficace, plus dynamique, ce passage de relais guidé rend cette dramaturgie véritablement active. Ainsi, relevant de la pensée rationnelle, l'ironie subsume le pulsionnel sans le faire disparaître, conformément au dessein de ne pas réduire l'hétérogène dans une fallacieuse unité. Elle conduit au final à rapporter tout ce que la dérision et le grotesque ont pu pointer à une critique de l'adhésion et de la croyance, véritable creuset des mécanismes de l'aliénation. Et de ce concept daté et idéologiquement connoté, le théâtre de Piemme invite à reprendre l'analyse en en désignant de nouvelles bases, de nouveaux 